\title{
Expectations of pregnant women for antenatal care services and factors affecting anxiety severity during the COVID-19 pandemic
}

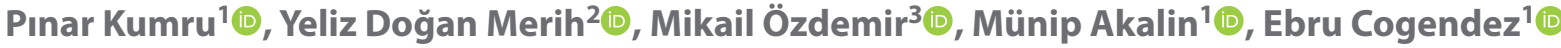 \\ ${ }^{1}$ University of Health Sciences, Zeynep Kamil Women and Children's Disease Training and Research Hospital, Istanbul, Turkey \\ ${ }^{2}$ University of Health Sciences Hamidiye Nursing Faculty/Turkey Institutes of Health Presidency, Istanbul, Turkey \\ ${ }^{3}$ Osmaniye Community Health Center, Osmaniye, Turkey
}

\begin{abstract}
Objectives: We aimed to evaluate the difficulties pregnant women encountered while receiving health care, their demands for antenatal care, and their mental state during the COVID-19 pandemic.

Material and methods: A total of 447 pregnant women were included in this cross-sectional study. The data were collected through a face-to-face questionnaire, which assessed participants' demographic, individual, and obstetric characteristics, their opinions regarding the COVID-19 pandemic, expectations from their antenatal care services, and their Beck Anxiety Inventory (BAl) scores.

Results: During the COVID-19 pandemic, it was determined that $17.2 \%$ of the pregnant women participating in our study could not go to antenatal follow-ups and almost half (45.9\%) demanded that their follow-ups be reduced due to the risk of coronavirus transmission. The BAI scores were found to be significantly higher in participants with low-income levels, chronic diseases, those in the third trimester, those with high-risk pregnancy either previous or current, and those who got pregnant unintentionally. Young age, unintentional conception, advanced pregnancy week, previous high-risk pregnancy, and failure to receive regular antenatal care were independent variables that predicted moderate-severe anxiety in logistic regression analysis.

Conclusions: In order to minimize the adverse effects of the COVID-19 pandemic on the mental health of pregnant women, it is important to develop support programs that contribute to the well-being of the mother and fetus by recognizing the pregnant women at risk in the antenatal period.
\end{abstract}

Key words: antenatal care; anxiety; Beck Anxiety Inventory; coronavirus; COVID-19; pandemic

Ginekologia Polska 2022; 93, 2: 142-150

\section{INTRODUCTION}

The 2019 Coronavirus Disease (COVID-19), which first emerged in the city of Wuhan, Hubei Province in China in December 2019, was declared a "Public Health Emergency of International Concern" on January 31, 2020, and as a pandemic on March 11, 2020 by the World Health Organization (WHO) [1].

Based on the limited data currently available related to COVID-19, there is no increased susceptibility or risk of severe morbidity and mortality among pregnant women compared to the non-pregnant women in the general population. However, viral diseases during pregnancy can cause adverse maternal and fetal outcomes. The association of COVID-19 with complications such as premature birth, fetal growth restriction, preeclampsia, gestational diabetes, hypertension, severe maternal disease, increased admission to the intensive care unit, and maternal death has been reported in the late pregnancy period (> 24 weeks) [2, 3]. Indeed, in a study by Zaigham and Andersson, it was reported that COVID-19 during pregnancy may be associated with severe maternal morbidity and the possibility of maternal-fetal transmission cannot be completely ruled out [4].

Based on the studies examining previous outbreaks, people are more likely to experience psychological problems

\section{Corresponding author:}

Pinar Kumru

University of Health Sciences, Zeynep Kamil Women and Children's Disease Training and Research Hospital, Istanbul, Turkey

e-mail:pkumru@gmail.com 
during public health emergencies, such as COVID-19. Pregnant women, who are one of the most susceptible, fragile, and vulnerable groups of the society, are expected to be affected by uncertainties regarding clinical impacts of this disease, decrease in support from families and friends due to isolation and quarantine, financial problems, and travel restrictions. It is noteworthy that especially outbreaks, pregnant women experience anxiety due to disruptions in their routine antenatal follow-ups, changes in health services (online meeting instead of face-to-face meeting, use of telemedicine, etc.), and difficulties in reaching the hospitals and physicians from which they receive care [5].

\section{Objectives}

in this study, we aimed to evaluate the difficulties pregnant women faced while receiving healthcare services during the COVID-19 pandemic, as well as changes in their lifestyle, their anxiety and to determine their expectations from prenatal care services.

\section{MATERIAL AND METHODS}

This study was carried out in the pregnancy follow-up outpatient clinics at the University of Health Sciences, Zeynep Kamil Women and Children Diseases Training and Research Hospital in Istanbul, Turkey between June-July 2020. During the pandemic, our hospital continued to serve women with low and high-risk pregnancies without any interruption by rearranging the working conditions.

\section{Population and sample}

The population of this cross-sectional study consisted of 447 pregnant women who applied to the pregnancy outpatient clinic of the hospital throughout the study period. All participants were between 19 and 45 years of age, were literate, had no communication problems, and gave consent to fill out the questionnaire upon being informed about the significance and objective of the study. Participants that had any clinical manifestations or symptoms of COVID-19, who were suspected of/diagnosed with COVID-19, and those previously diagnosed with any psychiatric disorder were excluded from the study.

The sample size was calculated through the analysis, which was conducted using the OpenEpi software (version 3). Based on the anxiety rate, the outcome of which was unpredictable and predicted as $50 \%$, a total of 377 participants were found to be sufficient with $5 \%$ alpha error and $99 \%$ power to represent the number of pregnant women (500) who applied to our hospital for the first time within one month.

\section{Data collection tools}

The data were collected through a questionnaire, which assessed participants' demographic, individual, and the obstetric characteristics, their opinions regarding the COVID-19 pandemic, expectations from their antenatal care services, and the Beck Anxiety Inventory (BAl) scores.

Pregnancy introduction form has been prepared in line with the literature. In this form, there were 6 sections that questioned the socio-demographic characteristics and medical history, obstetric history, changes in the lives of participants during the pandemic, their opinions about the COVID-19 pandemic, their concerns about COVID-19 infection, and the service they need during the pandemic [6-8]. Before the study, a preliminary version of the questionnaire was applied to a different set of pregnant women and the items of the questionnaire were revised accordingly. These patients that participated in the preliminary questionnaire were excluded from the study.

The participants' anxiety level and severity were assessed through the BAI, which was developed by Beck et al. [9] and adapted to Turkish by Ulusoy et al. [10]. It has been determined that the scale had an adequate reliability and validity $[11,12]$. BAl assesses the frequency of anxiety symptoms that are experienced by the individual. According to BAI, the scores are categorized as follows: 0-7 points indicate minimal anxiety, 8-15 points indicate mild anxiety, 16-25 points demonstrate moderate anxiety, and 26-63 points point to severe anxiety [10].

\section{Ethical statement}

Ethical approval for our study was obtained from the Ethics Committee of Health Sciences University, Zeynep Kamil Women and Children Diseases Training and Research Hospital (decision no 108, dated 03.06.2020). Before starting the survey, participants were informed that the survey was for research purposes and that their identities would be kept confidential within the scope of the confidentiality principle, and their written consents were obtained in this regard.

\section{Statistical methods}

The Statistical Package for the Social Sciences (SPSS Inc., version 17; Chicago, IL, USA) was used for statistical analyses. Data were expressed as numeric (\%) or mean \pm standard deviation (SD) and median (min-max) values where appropriate. Kolmogorov-Smirnov tests were performed for distribution of continuous data. Statistical analyses were performed by using Student t-test for normally distributed data and Mann-Whitney U test for non-normally distributed data. For categorical values, $\mathrm{p}$-values were calculated using the chi-square test (with Fisher exact test for groups with less than five subjects expected in a cell). The relationship between two sets of data was analyzed by Spearman's rank correlation test. Multivariate analysis was used for logistic regression analysis (Backward LR). P value of less than 0.05 was considered to show a statistically significant result. 


\section{RESULTS}

The mean age of the 447 pregnant women who participated in the study is $29.4 \pm 5.8$ years. The socio-demographic data of the participants are presented in detail in Table 1.

Evaluation of obstetric characteristics showed that the mean gravida was $2.39 \pm 1.40$ and mean week of gestation was $23.67 \pm 9.18$. It was found that $59.7 \%$ of the participants were multiparous, $20.6 \%$ experienced problems in their previous pregnancy, and $34 \%$ had a risk in their current pregnancy $(6.9 \%$ had thyroid disease, $6 \%$ had diabetes mellitus, 4.7\% had chronic $\mathrm{HT}, 4.3 \%$ had chronic respiratory disease and asthma) and $5.4 \%$ were smokers. It was determined that $82.1 \%$ of the participants attended antenatal follow-ups regularly during the pandemic, and $51.3 \%$ of those who did not participate in follow-ups attributed this to fear of being infected with the disease (Tab. 1).

Evaluation of the changes experienced by participants during the pandemic showed that $15.2 \%$ experienced shorter sleep duration and $57.5 \%$ experienced a decrease in their daily activities. It was determined that almost half of the participants (45.9\%) asked the health institutions to reduce the frequency of pregnancy follow-ups throughout the pandemic due to the concern of being infected with the disease, and the pregnant women who wanted to reduce the frequency of follow-ups were mostly in their first trimester (38\%) (Tab. 1).

In our study, it was determined that participants (73.8\%) were most frequently worried about the risk of transmission of COVID-19 from another patient during or after delivery at the hospital. The second most common concern (72.5\%) was that their babies would be harmed if they became infected with COVID-19 during pregnancy, followed by the worry that their spouse or relative could not be present during the delivery (68.7\%). In addition, nearly half (45.9\%) of the participants did not want to attend the follow-up because they were worried about being infected with the COVID-19 in the hospital setting, participants whose spouses were working were concerned that their spouses might infect them with COVID-19 (49.9\%), 75\% were unsure about breastfeeding during the pandemic or did not know if breastfeeding was safe. Moreover, $28.6 \%$ of the participants said they might opt for cesarean section instead of vaginal delivery, 33.8\% would increase interventions to hasten the delivery, $59.7 \%$ indicated that sufficient measurements were being taken in the hospital, and $79 \%$ of them thought that they may get infected with COVID-19 from healthcare staff in the hospital and that they were indecisive related to this issue (Tab. 2).

Among the participants, $95.3 \%$ requested designating isolated and clean areas for pregnant women to receive health care service in hospitals, $91.7 \%$ requested to be informed about the pregnancy follow-up process and screening tests during the pandemic, $90.6 \%$ requested to be examined by the appointment system to avoid contact while attending the pregnancy follow-ups. Also, $82.8 \%$ requested free examination and delivery services in private hospitals to reduce the volume during the pandemic, $87.9 \%$ requested to be informed about protective and preventive ways against COVID-19 infection, and $77.9 \%$ of them requested including psychological support in the health care services during the pandemic (Tab. 3).

When all the participants were evaluated, the mean BAI score was $13.25 \pm 11.27$. It was determined that BAl scores of those who had low-income levels, who had chronic diseases, who were in the third trimester, those at risk in the previous and current pregnancy, and those who become pregnant unintendedly were significantly higher $(p<0.05)$ (Tab. 4).

Among the participants, $39.6 \%$ had minimal anxiety, $24.2 \%$ had mild anxiety, $19.9 \%$ had moderate anxiety, and $16.3 \%$ had severe anxiety. Significantly higher level of moderate-severe anxiety was found in those who conceived unintentionally, those who had a problem in their previous and current pregnancy, those who were in the third trimester, did not receive regular antenatal follow-up ( $<<0.05$ ) (Tab. 5).

Logistic regression analysis (backward LR) was used to determine the target pregnancy group that independently affected the anxiety level and had a high risk of moderate to severe anxiety. While performing the multivariate analysis, variables that were significant in univariate analyzes and variables that were expected to be related according to the literature and may be confusing were included in the model. According to multivariate analysis, moderate-to-severe anxiety was less frequent in oldest participants [odds ratio (OR) 0.96; 95\% Cl 0.92-0.99; $\mathrm{p}=0.033$ ]. However, it was more frequent in those who conceived unintentionally (OR 2.02; $95 \% \mathrm{Cl} 1.17-3.50 ; \mathrm{p}=0.012)$, those in the later weeks of pregnancy (OR 1.09; 95\% Cl 1.02-1.18; $\mathrm{p}=0.016)$, those with high-risk pregnancies (OR 2.09; 95\% Cl 1.17-3.49; $p=0.012$ ), and those who did not have regular antenatal follow-ups (OR 2.51; 95\% Cl 1.41-4.48; $p=0.002$ ).

\section{DISCUSSION}

The COVID-19 pandemic [1] has had devastating effects all over the world. In most countries, health systems have faced collapse, and all elective surgeries and outpatient services, except emergency cases, have been partially or completely stopped. Each hospital implemented its own emergency action plan [13].

In a study conducted by Lebel et al. [14] with 1987 pregnant women, most of whom had high education and income levels, $89 \%$ of the participants stated that there were changes in pandemic-related antenatal care, and $90 \%$ of them stated that the person who was supposed to provide them with social support was not allowed at birth. In the same study, $35 \%$ of the pregnant women reported that they changed 
Table 1. Participants'socio-demographic, obstetric characteristics and distribution of changes experienced during the COVID-19 pandemic $(n=447)$

\section{Participants}

Age [years]

Duration of marriage [years]

Gravida

Parity

Week of gestation [weeks]

Mean age of their children [years]

Educational status

Working status during pandemic

Smoking habit

Social security status

Income of family

Family type

Chronic diseases

Unintended pregnancy status

Pregnancy trimester

\section{Multiparity}

Had problems during previous pregnancy

Risk in current pregnancy

Adequate knowledge of pregnancy, delivery and puerperium

Postpartum care training during pregnancy

Regular antenatal follow-ups

Reasons for not following $(n=80)$

Demand to reduce the number of pregnancy examinations during the pandemic

\section{For fear of getting infected}

Hospital could not serve due to pandemic

Couldn't find an appointment

Demand to reduce the number of examinations according to pregnancy trimester $(n=205)$

Sleep time in pregnancy during pandemic

Daily activity change during the pandemic

\begin{tabular}{|l|l|l|}
\hline $1^{\text {st }}$ trimester & 205 & 45.9 \\
\hline $2^{\text {nd }}$ trimester & 78 & 38.0 \\
\hline $3^{\text {rd }}$ trimester & 72 & 35.2 \\
\hline $4-6$ hours & 55 & 26.8 \\
\hline $7-8$ hours & 68 & 15.2 \\
\hline $9-10$ hours & 203 & 45.4 \\
\hline$\geq 11$ hours & 146 & 32.7 \\
\hline Decreased & 30 & 6.7 \\
\hline Not changed & 257 & 57.5 \\
\hline Increased & 169 & 37.8 \\
\hline
\end{tabular}

\section{mean \pm SD median (min-max)}

$29.4 \pm 5.8 \quad 29(19-44)$

$6.4 \pm 5.4 \quad 5(1-30)$

$2.4 \pm 1.40 \quad 2(1-9)$

$1.0 \pm 1.0 \quad 1(0-6)$

$23.7 \pm 9.2 \quad 29(26-39)$

$5.9 \pm 3.9 \quad 5(1-28)$

$\mathrm{n} \quad \%$

\begin{tabular}{l|l}
94 & 21.0
\end{tabular}

\begin{tabular}{l|l}
108 & 24.2 \\
\hline
\end{tabular}

\begin{tabular}{l|l}
125 & 28.0
\end{tabular}

\begin{tabular}{l|l}
120 & 26.8 \\
\hline
\end{tabular}

\begin{tabular}{l|l|}
17 & 3.8 \\
\hline
\end{tabular}

\begin{tabular}{l|l}
38 & 8.5
\end{tabular}

\begin{tabular}{l|l}
44 & 9.6
\end{tabular}

\begin{tabular}{l|l|l}
\hline 349 & 78.1
\end{tabular}

\begin{tabular}{l|l}
\hline 24 & 5.4 \\
\hline
\end{tabular}

\begin{tabular}{l|l|l}
\hline 387 & 86.6 \\
\hline
\end{tabular}

\begin{tabular}{l|l}
36 & 8.1
\end{tabular}

\begin{tabular}{l|l}
364 & 81.4
\end{tabular}

\begin{tabular}{l|l|l}
188 & 42.1
\end{tabular}

\begin{tabular}{l|l}
238 & 53.2
\end{tabular}

\begin{tabular}{l|l}
21 & 4.7
\end{tabular}

\begin{tabular}{l|l|l}
\hline 376 & 84.1 \\
\hline
\end{tabular}

\begin{tabular}{l|l|l}
71 & 15.9
\end{tabular}

\begin{tabular}{l|l}
101 & 22.6
\end{tabular}

\begin{tabular}{l|l}
89 & 19.9
\end{tabular}

\begin{tabular}{l|l|l}
\hline 145 & 32.4
\end{tabular}

\begin{tabular}{l|l|l}
146 & 32.7
\end{tabular}

\begin{tabular}{l|l|l}
156 & 34.9
\end{tabular}

\begin{tabular}{l|l|l}
267 & 59.7
\end{tabular}

\begin{tabular}{l|l|}
92 & 20.6
\end{tabular}

\begin{tabular}{l|l}
152 & 34.0
\end{tabular}

\begin{tabular}{l|l}
322 & 72.0
\end{tabular}

\begin{tabular}{l|l}
150 & 33.6 \\
\hline
\end{tabular}

\begin{tabular}{|l|l|}
367 & 82.1
\end{tabular}

\begin{tabular}{l|l}
41 & 51.3
\end{tabular}

\begin{tabular}{l|l}
17 & 21.3
\end{tabular}

\begin{tabular}{l|l}
22 & 27.5
\end{tabular}


Table 2. Participants' concerns about COVID-19 infection $(n=447)$

\begin{tabular}{|c|c|c|c|c|c|c|}
\hline & \multicolumn{2}{|l|}{ Yes } & \multicolumn{2}{|c|}{ Undecided } & \multicolumn{2}{|l|}{ No } \\
\hline & n & $\%$ & n & $\%$ & n & $\%$ \\
\hline I do not want to go to follow-ups because I might get COVID-19 infection from hospitals. & 205 & 45.9 & 145 & 32.4 & 97 & 21.7 \\
\hline I think that enough precautions are being taken at the hospital where I go for antenatal care. & 267 & 59.7 & 137 & 30.6 & 43 & 9.6 \\
\hline I think the disinfectants I use during pregnancy will harm my baby. & 100 & 22.4 & 175 & 39.1 & 172 & 38.5 \\
\hline I think that COVID-19 infection can be transmitted from healthcare professionals. & 161 & 36.0 & 192 & 43.0 & 94 & 21.0 \\
\hline My husband is working and I'm afraid he might bring home COVID-19 infection. & 223 & 49.9 & 95 & 21.3 & 129 & 28.9 \\
\hline I think my baby will suffer if I get COVID-19 infection during pregnancy. & 324 & 72.5 & 93 & 20.8 & 30 & 6.7 \\
\hline $\begin{array}{l}\text { I think that even if there is no infection, there will be a risk of transmission from another } \\
\text { patient at the hospital or after birth. }\end{array}$ & 330 & 73.8 & 95 & 21.3 & 22 & 4.9 \\
\hline I think there will be increase number of interventions in this period to accelerate birth. & 151 & 33.8 & 167 & 37.4 & 129 & 28.9 \\
\hline I'm afraid of not getting physical/emotional support during delivery. & 198 & 44.3 & 139 & 31.1 & 110 & 24.6 \\
\hline It worries me that my husband or a relative will not be there during the delivery. & 307 & 68.7 & 60 & 13.4 & 80 & 17.9 \\
\hline Experiencing pain while wearing a mask constantly worries me. & 289 & 64.7 & 77 & 17.2 & 81 & 18.1 \\
\hline I think I will be referred to a planned cesarean section instead of a vaginal delivery. & 128 & 28.6 & 119 & 26.6 & 200 & 44.7 \\
\hline If I am COVID-19 positive, I think I can breastfeed my baby. & 136 & 30.4 & 152 & 34.0 & 159 & 35.6 \\
\hline I am worried about going to the healthcare facility for my baby's vaccinat & 223 & 49.9 & 99 & 22.1 & 125 & 28.0 \\
\hline
\end{tabular}

Table 3. The subjects that participants want to be included in the service processes during the COVID-19 pandemic $(n=447)$

\begin{tabular}{|c|c|c|c|c|c|c|}
\hline & \multicolumn{2}{|l|}{ Yes } & \multicolumn{2}{|c|}{ Undecided } & \multicolumn{2}{|l|}{ No } \\
\hline & $\mathbf{n}$ & $\%$ & $\mathbf{n}$ & $\%$ & $\mathbf{n}$ & $\%$ \\
\hline Designation of isolated and sterile areas in hospitals for pregnant women to receive service & 426 & 95.3 & 18 & 4.0 & 3 & 0.7 \\
\hline $\begin{array}{l}\text { Providing examination by appointment system due to the least need for contact while going to } \\
\text { pregnancy controls }\end{array}$ & 405 & 90.6 & 36 & 8.1 & 6 & 1.3 \\
\hline Continuing pregnancy education through online classes & 304 & 68.0 & 110 & 24.6 & 33 & 7.4 \\
\hline Providing consultations and care services for pregnant women via telemedicine and online system & 335 & 74.9 & 89 & 19.9 & 23 & 5.1 \\
\hline $\begin{array}{l}\text { Performing pregnancy follow-ups in primary care family health centers to reduce the density in } \\
\text { hospitals during the pandemic }\end{array}$ & 326 & 72.9 & 92 & 20.6 & 29 & 6.5 \\
\hline $\begin{array}{l}\text { During the pandemic, pregnancy follow-ups were carried out as home visits to reduce the density in } \\
\text { hospitals }\end{array}$ & 225 & 50.3 & 122 & 27.3 & 100 & 22.3 \\
\hline $\begin{array}{l}\text { Providing free examination and delivery services in private hospitals to reduce the patient volume } \\
\text { during pandemic periods }\end{array}$ & 370 & 82.8 & 53 & 11.9 & 24 & 5.4 \\
\hline Informing about COVID-19 infection control and prevention methods & 393 & 87.9 & 29 & 6.5 & 25 & 5.6 \\
\hline Informing about pregnancy follow-up process and screening tests during pandemic periods & 410 & 91.7 & 18 & 4.0 & 19 & 4.3 \\
\hline Informing about pregnancy follow-up process and screening tests during pandemic periods & 348 & 77.9 & 68 & 15.2 & 31 & 6.9 \\
\hline
\end{tabular}

birth plans such as location, social support and childcare due to the pandemic, $74 \%$ had problems in accessing other health services during pregnancy, and $9 \%$ could not access psychological counseling services. In our study, $82.1 \%$ of the participants went to antenatal follow-ups regularly during the pandemic. However, $51.3 \%$ of the participants who did not go to antenatal follow-up did not do so due to fear of being infected. The fact that most of the pregnant women participating in the study received antenatal care may be related to the uninterrupted service of our hospital during the pandemic. In our study, most of the participants stated that they preferred practices that were revised in accordance with the preventive measures of the pandemic and required less contact, instead of the existing practices in antenatal care processes during the COVID-19 pandemic period. In addition, $45.9 \%$ of the participants asked health institutions to reduce the frequency of their pregnancy follow-ups due to the concern of COVID-19 transmission and $38 \%$ of those who wanted to reduce the frequency of follow-up were in their first trimester.

In another study conducted by Akgör et al. [15] with 297 pregnant women, more than half of the participants 
Table 4. Factors affecting participants' Beck anxiety scale score $(n=447)$

\begin{tabular}{|c|c|c|c|c|c|}
\hline & & \multicolumn{3}{|c|}{ Beck anxiety scale score } & \multirow{2}{*}{$p$ value } \\
\hline & & Mean & (SD) & Median & \\
\hline \multirow{4}{*}{ Education status } & Elementary school & 13.14 & 11.40 & 10.00 & \multirow{4}{*}{0.697} \\
\hline & Secondary school & 12.76 & 11.23 & 9.50 & \\
\hline & High school & 14.17 & 11.58 & 12.00 & \\
\hline & University & 12.82 & 10.97 & 9.00 & \\
\hline \multirow{2}{*}{ Working status } & Not working & 13.28 & 11.18 & 10.00 & \multirow{2}{*}{0.786} \\
\hline & Working & 13.14 & 11.64 & 10.00 & \\
\hline \multirow{4}{*}{ Working status during pandemic } & Formal & 11.47 & 12.26 & 9.00 & \multirow{4}{*}{0.065} \\
\hline & Flexible work & 13.50 & 12.93 & 8.50 & \\
\hline & Work from home & 11.65 & 9.77 & 9.50 & \\
\hline & Others & 24.00 & 9.35 & 23.00 & \\
\hline \multirow{4}{*}{ Education status of spouse } & Elementary school & 11.89 & 9.78 & 9.00 & \multirow{4}{*}{0.218} \\
\hline & Secondary school & 12.24 & 11.80 & 9.00 & \\
\hline & High school & 14.63 & 11.78 & 11.00 & \\
\hline & University & 13.23 & 10.79 & 11.00 & \\
\hline \multirow{3}{*}{ Income level } & Low & 14.95 & 12.02 & 12.00 & \multirow{3}{*}{$0.038^{*}$} \\
\hline & Medium & 12.14 & 10.63 & 9.00 & \\
\hline & High & 10.57 & 9.77 & 11.00 & \\
\hline \multirow{2}{*}{ Chronic diseases } & Present & 15.58 & 12.12 & 13.00 & \multirow{2}{*}{$0.028^{*}$} \\
\hline & Absent & 12.57 & 10.94 & 10.00 & \\
\hline \multirow{3}{*}{ Pregnancy trimester } & 1st trimester & 8.70 & 8.07 & 7.00 & \multirow{3}{*}{$<0.001^{*}$} \\
\hline & 2nd trimester & 9.23 & 8.51 & 7.00 & \\
\hline & 3rd trimester & 21.24 & 11.77 & 21.00 & \\
\hline \multirow{2}{*}{ Parity } & Nullipar & 12.75 & 11.79 & 9.00 & \multirow{2}{*}{0.195} \\
\hline & Multipar & 13.58 & 10.92 & 11.00 & \\
\hline \multirow{2}{*}{ Had problems during previous pregnancy } & Yes & 15.99 & 12.40 & 14.00 & \multirow{2}{*}{$0.025^{*}$} \\
\hline & No & 12.58 & 10.94 & 9.00 & \\
\hline \multirow{2}{*}{ Risk in current pregnancy } & Present & 15.76 & 12.39 & 14.00 & \multirow{2}{*}{$<0.001 *$} \\
\hline & Absent & 11.96 & 10.44 & 9.00 & \\
\hline \multirow{2}{*}{ Intended pregnancy } & Intended & 12.15 & 11.00 & 9.00 & \multirow{2}{*}{ 0.003* } \\
\hline & Unintended & 17.65 & 11.32 & 17.00 & \\
\hline \multirow{2}{*}{ Postpartum care training during pregnancy } & Present & 12.11 & 10.95 & 9.00 & \multirow{2}{*}{0.126} \\
\hline & Absent & 13.82 & 11.41 & 11.00 & \\
\hline
\end{tabular}

*Statistically significant at $p<0.05$

were concerned about delaying their appointments and not reaching their specialists, despite having been provided with uninterrupted health care during the COVID-19 pandemic. The authors reported that this situation could be related to the probability of health system collapse and disinformation on social media.

Xian et al. [16] reported that regular physical activity during pregnancy had a protective effect on anxiety and depression. Indeed, in the study of Kahyaoğlu et al. [17] it was reported that the risk of anxiety and depression increased in pregnant women who did not engage in regular physical activity. In our study, it was determined that the daily activities of $57.5 \%$ of the participants decreased due to the social isolation and quarantine practices applied in the pandemic.

In our study, $72.5 \%$ of participants worried that their baby may be harmed when infected with COVID-19. Moreover, $68.7 \%$ of pregnant women were concerned that their spouse or a relative will not be with them at birth. As a matter of fact, approximately one third participants stated that they were worried about interventions that would accelerate labor, while $28.6 \%$ of them stated that they could be referred to a planned cesarean in the management of their 
Table 5. Factors affecting participants' Beck anxiety level $(n=447)$

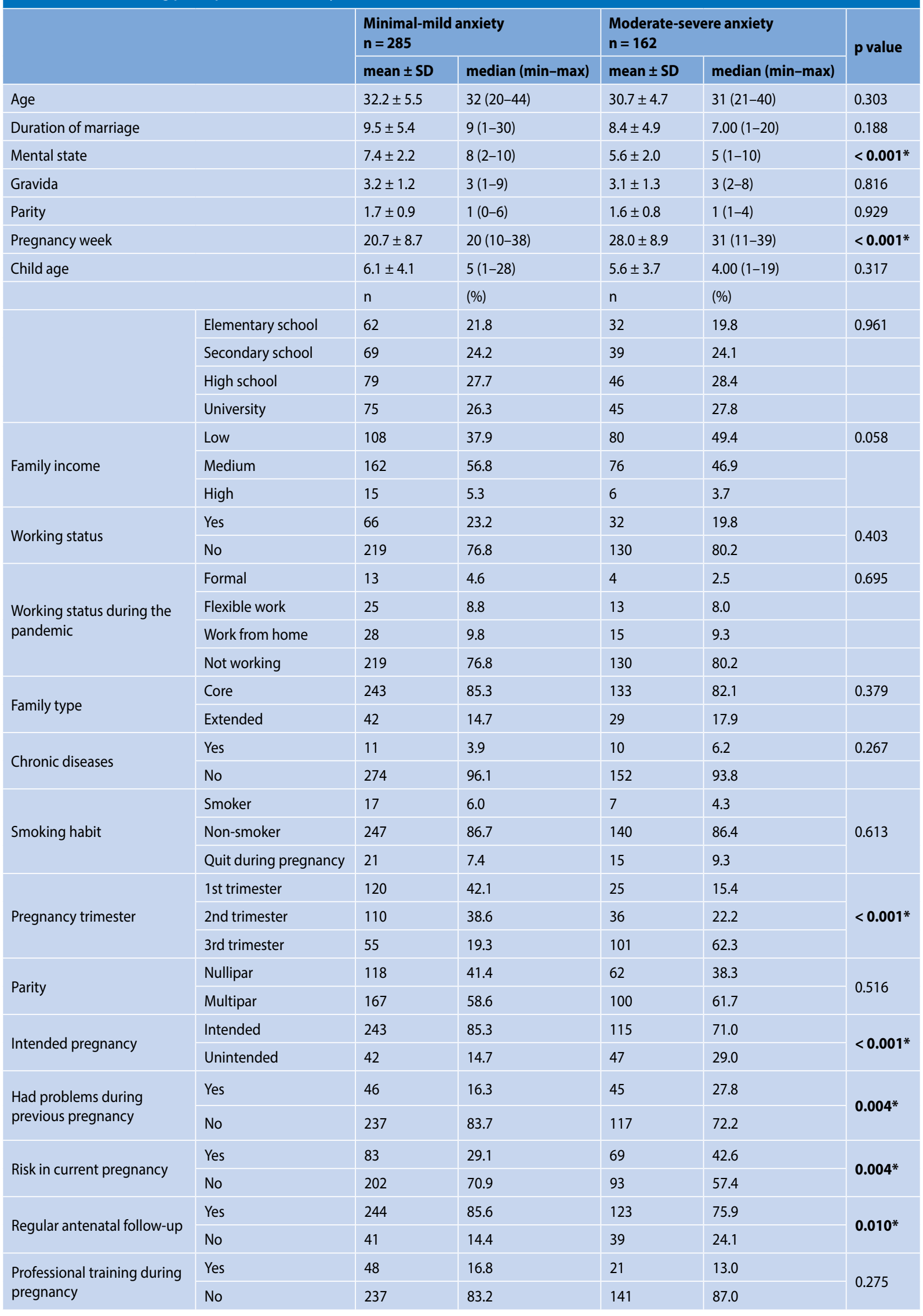

*Statistically significant at $p<0.05$ 
deliveries. In the study by Xian et al. [16], it was reported that $12.8 \%$ of pregnant women wanted to perform a planned cesarean section instead of waiting for a hospital birth.

Fetal health is one of the main concerns of the expectant mother during pregnancy. In the study by Ahorsu et al. [18], it was shown that pregnant women felt fear and anxiety about fetal and neonatal health during the pandemic. It has been reported that these pregnant women want to terminate their pregnancy early or deliver via cesarean section due to the stress and anxiety caused by the risk of transmission. Uncertainty regarding the duration of the COVID-19 pandemic is another factor that increases the anxiety level of pregnant women [19].

There are data showing that prenatal anxiety and depression affect maternal and infant health both physically and psychologically in the short and long term. Among these, an increased risk of abortion, preterm delivery, low birth weight, low Apgar score at birth, and long-term cognitive and behavioral problems in the mothers themselves and their children have been reported [20-23].

Studies have reported higher levels of anxiety and depression in pregnant women compared to non-pregnant women $[24,25]$. In studies conducted before the COVID-19 pandemic, 5-13\% anxiety, 4-15\% depression and $0.9-3.8 \%$ combination of anxiety and depression were reported in pregnant women [26-28]. During the pandemic, the prevalence of anxiety in pregnant women has been reported to vary between $63 \%$ and $68 \%[14,29,30]$. In the study by Kahyaoğlu and Küçükkaya [17], this rate was found to be $64.5 \%$ and $56.3 \%$ for anxiety and depression, respectively. As a result of the study, the authors reported that low education level, lack of regular physical activity, having to make face-to-face hospital visits and not having enough information about the effects of COVID-19 on pregnancy were the most important factors associated with the development of anxiety and depression in pregnant women.

In our study, we found moderate and severe anxiety levels as $19.9 \%$ and $16.3 \%$, respectively. Multivariate analysis revealed that moderate-severe anxiety was associated with unintended pregnancy, previous or current high-risk pregnancy, not having regular prenatal follow-up, and being in the third trimester. We also found that our patients had similar anxiety levels compared to other studies [14, 29,30]. However, we think that the lower levels of moderate and severe anxiety in our participants are related to integrated health services, effective information and family supports during pregnancy.

This study is one of the leading researches that identified the levels of concern, demand, and anxiety of pregnant women regarding the COVID-19 pandemic in Turkey. Most of the previous COVID-19-related survey studies were performed with participants who use social media and the inter- net and those who had a low-risk pregnancy, higher income, and educational status. Furthermore, online surveys have less likelihood of being responded to. The strengths of our study were that we conducted our surveys face-to-face and thus we were able to reach individuals with low/medium education and income rates, which are difficult to access online. Moreover, we were able to reach individuals with high-risk pregnancies. Hence, we believe that our study will help establish a scientific basis for development of health policies that will optimize maternal and infant health by determining the health needs of various pregnancy groups.

However, our study has some limitations. The cross-sectional nature of survey research is one of the primary limitations of this study. Another limitation is that the study was performed solely with literate pregnant women who applied to our hospital during a limited period. Thus, obtained data may not be generalizable to the entire population of Turkey. Another limitation is that the information on COVID-19 has not been fully verified yet, and scientific data and information are updated continuously.

\section{CONCLUSIONS}

The detection of anxiety, albeit minimal, in the majority of pregnant women participating in the study once again demonstrated the importance of exposure to stress factors during the pandemic. In order to minimize the adverse effects of COVID-19 pandemic on the mental health of pregnant women, it is important to develop support programs that will contribute to the well-being of the mother and fetus by recognizing those at risk in the antenatal period.

\section{Conflict of interest}

The authors have stated explicitly that there are no conflicts of interest in connection with this article.

\section{Funding}

This research received no specific grant from any funding agency in the public, commercial, or not-for-profit sectors.

\section{REFERENCES}

1. Cucinotta D, Vanelli M. WHO declares COVID-19 a pandemic. Acta Biomed. 2020; 91(1): 157-160, doi: 10.23750/abm.v91i1.9397, indexed in Pubmed: 32191675.

2. Buonsenso D, Raffaelli F, Tamburrini $E$, et al. Clinical role of lung ultrasound for diagnosis and monitoring of COVID-19 pneumonia in pregnant women. Ultrasound Obstet Gynecol. 2020; 56(1): 106-109, doi: 10.1002/uog.22055, indexed in Pubmed: 32337795.

3. Liu W, Wang J, Li W, et al. Clinical characteristics of 19 neonates born to mothers with COVID-19. Front Med. 2020; 14(2): 193-198, doi: 10.1007/s11684-020-0772-y, indexed in Pubmed: 32285380.

4. Zaigham M, Andersson O. Maternal and perinatal outcomes with COVID-19: A systematic review of 108 pregnancies. Acta Obstet Gynecol Scand. 2020; 99(7): 823-829, doi: 10.1111/aogs.13867, indexed in Pubmed: 32259279.

5. Cheng C, Cheung MWL. Psychological responses to outbreak of severe acute respiratory syndrome: a prospective, multiple time-point study. J Pers. 2005; 73(1): 261-285, doi: 10.1111/j.1467-6494.2004.00310.x indexed in Pubmed: 15660679. 
6. Breslin N, Baptiste C, Gyamfi-Bannerman C, et al. Coronavirus disease 2019 infection among asymptomatic and symptomatic pregnant women: two weeks of confirmed presentations to an affiliated pair of New York City hospitals. Am J Obstet Gynecol MFM. 2020; 2(2): 100118, doi: 10.1016/j.ajogmf.2020.100118, indexed in Pubmed: 32292903.

7. Wong S, Chow K, Leung T, et al. Pregnancy and perinatal outcomes of women with severe acute respiratory syndrome. Am J Obstet Gynecol. 2004; 191(1): 292-297, doi: 10.1016/j.ajog.2003.11.019, indexed in Pubmed: 15295381.

8. Siston AM, Rasmussen SA, Honein MA, et al. Pandemic H1N1 Influenza in Pregnancy Working Group. Pandemic 2009 influenza A(H1N1) virus illness among pregnant women in the United States. JAMA. 2010; 303(15): 1517-1525, doi: 10.1001/jama.2010.479, indexed in Pubmed: 20407061.

9. Beck AT, Epstein N, Brown G, et al. An inventory for measuring clinical anxiety: psychometric properties. J Consult Clin Psychol. 1988; 56(6): 893-897, doi: 10.1037//0022-006x.56.6.893, indexed in Pubmed: 3204199.

10. Ulusoy M, Sahin NH, Erkmen H. The Beck anxiety inventory: psychometric properties. J Cogn Psychother. 1998; 12(2): 163-172.

11. Wang C, Pan R, Wan X, et al. Immediate psychological responses and associated factors during the initial stage of the 2019 coronavirus disease (COVID-19) epidemic among the general population in China. Int J Environ Res Public Health. 2020; 17(5): 1729, doi: 10.3390/ijerph17051729, indexed in Pubmed: 32155789.

12. Kang YT, Yao Y, Dou J, et al. Prevalence and risk factors of maternal anxiety in late pregnancy in china. Int J Environ Res Public Health. 2016; 13(5): 468, doi: 10.3390/ijerph13050468, indexed in Pubmed: 27153080.

13. Centers for Disease Control and Prevention. Coronavirus disease 2019 (COVID-19). US Department of Health and Human Services. 2020. https:// www.cdc.gov/coronavirus/2019-ncov/transmission/ (2021-02-26).

14. Lebel C, MacKinnon A, Bagshawe $M$, et al. Elevated depression and anxiety symptoms among pregnant individuals during the COVID-19 pandemic. J Affect Disord. 2020; 277: 5-13, doi: 10.1016/j.jad.2020.07.126, indexed in Pubmed: 32777604.

15. Akgor U, Fadıloglu E, Soyak B, et al. Anxiety, depression and concerns of pregnant women during the COVID-19 pandemic. Arch Gynecol Obstet. 2021; 304(1): 125-130, doi: 10.1007/s00404-020-05944-1, indexed in Pubmed: 33433702.

16. Tang X, Lu Z, Hu D, et al. Influencing factors for prenatal Stress, anxiety and depression in early pregnancy among women in Chongqing, China. J Affect Disord. 2019; 253: 292-302, doi: 10.1016/j.jad.2019.05.003, indexed in Pubmed: 31077972.

17. Kahyaoglu Sut $\mathrm{H}$, Kucukkaya B. Anxiety, depression, and related factors in pregnant women during the COVID-19 pandemic in Turkey: a web-based cross-sectional study. Perspect Psychiatr Care. 2021; 57(2): 860-868, doi: 10.1111/ppc.12627, indexed in Pubmed: 32989798.

18. Ahorsu DK, Lin CY, Imani V, et al. The fear of COVID-19 scale: development and initial validation. Int J Ment Health Addict. 2020 [Epub ahead of print]: 1-9, doi: 10.1007/s11469-020-00270-8, indexed in Pubmed: 32226353.

19. Taubman-Ben-Ari O, Chasson M, Abu Sharkia S, et al. Distress and anxiety associated with COVID-19 among Jewish and Arab pregnant women in Israel. J Reprod Infant Psychol. 2020; 38(3): 340-348, doi: 10.1080/02646838.2020.1786037, indexed in Pubmed: 32573258.

20. Grigoriadis S, Graves L, Peer M, et al. Maternal anxiety during pregnancy and the association with adverse perinatal outcomes: systematic review and meta-analysis. J Clin Psychiatry. 2018; 79(5): 17r12011, doi: 10.4088/JCP.17r12011, indexed in Pubmed: 30192449.

21. Alder J, Fink N, Bitzer J, et al. Depression and anxiety during pregnancy: a risk factor for obstetric, fetal and neonatal outcome? A critical review of the literature. J Matern Fetal Neonatal Med. 2007; 20(3): 189-209, doi: 10.1080/14767050701209560, indexed in Pubmed: 17437220.

22. Kinsella MT, Monk C. Impact of maternal stress, depression and anxiety on fetal neurobehavioral development. Clin Obstet Gynecol. 2009; 52(3): 425-440, doi: 10.1097/GRF.0b013e3181b52df1, indexed in Pubmed: 19661759.

23. Kajdy A, Feduniw S, Ajdacka U, et al. Risk factors for anxiety and depression among pregnant women during the COVID-19 pandemic: A web-based cross-sectional survey. Medicine (Baltimore). 2020; 99(30): e21279, doi: 10.1097/MD.0000000000021279, indexed in Pubmed: 32791708.

24. Chen Yu, Li Z, Zhang YY, et al. Maternal health care management during the outbreak of coronavirus disease 2019. J Med Virol. 2020; 92(7): 731-739, doi: 10.1002/jmv.25787, indexed in Pubmed: 32219871.

25. Durankuş F, Aksu E. Effects of the COVID-19 pandemic on anxiety and depressive symptoms in pregnant women: a preliminary study. J Matern Fetal Neonatal Med. 2020 [Epub ahead of print]: 1-7, doi: 10.1080/14767058.2020.1763946, indexed in Pubmed: 32419558.

26. Brunton RJ, Dryer R, Saliba A, et al. Pregnancy anxiety: a systematic review of current scales. J Affect Disord. 2015; 176: 24-34, doi: 10.1016/j. jad.2015.01.039, indexed in Pubmed: 25687280.

27. Sinesi $A$, Maxwell M, O'Carroll R, et al. Anxiety scales used in pregnancy: systematic review. BJPsych Open. 2019; 5(1): e5, doi: 10.1192/bjo.2018.75, indexed in Pubmed: 30762504.

28. Vameghi R, Akbari SA, Sajjadi $H$, et al. Path analysis of the association between socio-economic status, anxiety, perceived stress, social support and women's depression. International Journal of Women's Health and Reproduction Sciences. 2018;6(3): 283-289, doi: 10.15296/ijwhr.2018.47.

29. Saccone G, Florio A, Aiello F, et al. Psychological impact of coronavirus disease 2019 in pregnant women. Am J Obstet Gynecol. 2020; 223(2): 293-295, doi: 10.1016/j.ajog.2020.05.003, indexed in Pubmed: 32387321.

30. Corbett GA, Milne SJ, Hehir MP, et al. Health anxiety and behavioural changes of pregnant women during the COVID-19 pandemic. Eur J Obstet Gynecol Reprod Biol. 2020; 249: 96-97, doi: 10.1016/j. ejogrb.2020.04.022, indexed in Pubmed: 32317197. 